\title{
GENERALIZED WRIGHT FUNCTION AND ITS PROPERTIES USING EXTENDED BETA FUNCTION
}

\author{
NABIULLAH KHAN, TALHA USMAN AND MOHD AMAN
}

\begin{abstract}
In this paper, we introduce a new generalization of the Wright function by using an extended beta function and study some classical properties of this function. We establish several formulas involving integral transforms (e.g. Jacobi transform, Gegenbauer transform) and the generalized family of Wright function that does not seem to be reported in the literature even for the basic Wright function. Furthermore, we discuss other results including the recurrence relation, derivative formula, fractional derivative formula and also a partly bilateral and partly unilateral generating relation for the generalized Wright function.
\end{abstract}

\section{Introduction and preliminaries}

The special functions of mathematical physics are found to be very useful in finding the solutions of initial and boundary value problems associated with partial differential equations and fractional differential equations. Special functions have widespread applications in areas of engineering as well, and often new perspectives in special functions are motivated by such connections. Several special functions, called now, special functions of fractional calculus play a very interesting role in the solution of fractional order differential equations such as Mittag-Leffler function, Wright function, and its auxiliary functions and Fox $H$ - function. The Wright function was first introduced [21, 22, 23, 24] during the study of the asymptotic theory of partitions of natural numbers. It plays an important role in the solution of linear partial differential equations and subsequently, many other applications for instance, in the Mikusiński's operational calculus ([7], see also [8]) and in the theory of Hankel type integral transforms have been found. Recently, this function has appeared in the area related to the partial differential equation of fractional order. Considering the boundary value problems for the fractional diffusion-wave equation, that is, the linear partial integro-differential equation

2010 Mathematics Subject Classification. 33B15, 33C10, 33C15, 33E12, 33E50, 44A15.

Key words and phrases. Beta function, Generalized Beta function, Wright function, Generalized Wright function, Mellin transform, Riemann-Liouville fractional derivative, Fox-Wright function and Gamma function.

Corresponding author: Nabiullah Khan. 
obtained from the classical diffusion or wave equation by replacing the first or second-order time derivatives by a fractional derivative of order $\alpha$ with $0<\alpha<2$, it was found the corresponding functions can be represented in terms of the Wright function. In this sequel, we intend to introduce a new generalization of Wright function by using the known extended beta function.

Let $\mathbb{C}, \mathbb{R}^{+}, \mathbb{N}$, and $\mathbb{Z}_{0}^{-}$be the sets of complex numbers, positive real numbers, positive integers, and non-positive integers, respectively, and let $\mathbb{R}_{0}^{+}:=\mathbb{R}^{+} \cup\{0\}$ and $\mathbb{N}_{0}:=\mathbb{N} \cup\{0\}$.

We recall the classical Wright function $W_{\alpha, \beta}(z)[8,14]$ defined by the series representation

$$
W_{\alpha, \beta}(z)=\sum_{n=0}^{\infty} \frac{z^{n}}{n !} \frac{1}{\Gamma(\alpha n+\beta)} \quad(\beta \in \mathbb{C}, \alpha>-1) .
$$

The two suplementary functions ([5]; see also $[8,14,15])$ of the above function are given as

$$
\begin{array}{ll}
M_{\alpha}(z)=W_{-\alpha, 1-\alpha}(-z)=\sum_{n=0}^{\infty} \frac{(-1)^{n} z^{n}}{n ! \Gamma(1-\alpha(n+1))} & (0<\alpha<1), \\
F_{\alpha}(z)=W_{-\alpha, 0}(-z)=\sum_{n=1}^{\infty} \frac{(-1)^{n}}{\Gamma(-\alpha n)} \frac{z^{n}}{n !} & (0<\alpha<1) .
\end{array}
$$

Recently, for real $\alpha$ and $\beta, \gamma, \delta \in \mathbb{C} ; \alpha>-1, \delta \neq 0,-1,-2, \ldots$ with $z \in \mathbb{C}$ and $|z|<1$ with $\alpha=-1$, Moustafa et al. [5] defined the generalization of the Wright function as

$$
W_{\alpha, \beta}^{\gamma, \delta}(z)=\sum_{n=0}^{\infty} \frac{z^{n}(\gamma)_{n}}{n !(\delta)_{n} \Gamma(\alpha n+\beta)},
$$

where

$$
\frac{\Gamma(\gamma+n)}{\Gamma(\gamma)}=(\gamma)_{n}=\gamma(\gamma+1)(\gamma+2) \cdots(\gamma+n-1) \text {. }
$$

is a pochhammer symbol (see, [18, p.2 and pp.4-6]) and $\Gamma(\cdot)$ is the gamma function (see, [18, Section 1.1]). The two auxillary functions (generalized Wright type) for all complex variable $z \neq 0$ and for any order $\alpha \in(0,1)$ were defined as

$$
\begin{aligned}
& M_{\alpha}^{\gamma, \delta}(z)=W_{-\alpha, 1-\alpha}^{\gamma, \delta}(-z)=\sum_{n=0}^{\infty} \frac{(-1)^{n} z^{n}}{n !} \frac{(\gamma)_{n}}{\Gamma(1-\alpha(n+1))(\delta)_{n}}, \\
& F_{\alpha}^{\gamma, \delta}(z)=W_{-\alpha, 0}^{\gamma, \delta}(-z)=\sum_{n=1}^{\infty} \frac{(\gamma)_{n}}{\left.(\delta)_{n} \Gamma(-\alpha n)\right)} \frac{(-1)^{n} z^{n}}{n !} .
\end{aligned}
$$

An extension of Euler's beta function ([2]; see also, [13, p.113(1.2)]) introduced by Chaudhry et al. is defined as

$$
\begin{gathered}
B_{p}(x, y)=\int_{0}^{1}(1-t)^{y-1} t^{x-1} e^{-\frac{p}{(1-t) t}} d t \\
(\min \{\Re(p), \Re(x), \Re(y)\}>0),
\end{gathered}
$$

and $B(x, y)(p=0)$ is the well-known beta function (see, [18, Section 1.1]). 
Further, a more generalized form of extended beta function [16] is defined as

$$
\begin{aligned}
B_{p}^{c, d}(x, y)= & \int_{0}^{1}(1-t)^{y-1} t^{x-1}{ }_{1} F_{1}\left[c ; d ;-\frac{p}{(1-t) t}\right] d t \\
& \left(\min \{\Re(y), \Re(x)\}>0 ; p \in \mathbb{R}_{0}^{+}\right) .
\end{aligned}
$$

Srivastava et al. [19] presented the following generalized beta function

$$
\begin{aligned}
B^{\left(\left\{v_{m}\right\}_{m \in \mathbb{N}_{0}}\right)}(x, y ; p) & =\int_{0}^{1}(1-t)^{y-1} t^{x-1} \mathscr{F}\left[\left\{v_{m}\right\}_{m \in \mathbb{N}_{0}} ;-\frac{p}{(1-t) t}\right] d t \\
& \left(\min \{\Re(y), \Re(x)\}>0 ; p \in \mathbb{R}_{0}^{+}\right),
\end{aligned}
$$

where, $\mathscr{F}\left(\left\{v_{m}\right\}_{m \in \mathbb{N}_{0}} ; z\right)$ is the function, analytic within the disk $|z|<\Re \quad(0<\Re<\infty)$ and its Taylor-Maclaurin coefficients be explicitly denoted by the sequence $\left\{v_{m}\right\}_{m \in \mathbb{N}_{0}}$.

The classical Jacobi polynomials $P_{n}^{(\alpha, \beta)}(z)$ (see, e.g., [4, Chapter 10] and [20, Chapter 4]) in terms of hypergeometric function are defined as

$$
P_{n}^{(\alpha, \beta)}(z)=\left(\begin{array}{c}
\alpha+n \\
n
\end{array}\right){ }_{2} F_{1}\left[\begin{array}{c}
-n, \alpha+\beta+n+1 ; \frac{1-z}{2} \\
\alpha+1 ;
\end{array}\right]
$$

where, ${ }_{2} F_{1}$ is the familiar Gauss hypergeometric function.

The Jacobi transform formula for a function $f(z)$ is defined as

$$
\begin{gathered}
\mathbb{J}^{\alpha, \beta}[f(z) ; n]=\int_{-1}^{1}(1-z)^{\alpha}(1+z)^{\beta} P_{n}^{(\alpha, \beta)}(z) f(z) d z \\
\left(\min \{\Re(\alpha), \Re(\beta)\}>-1 ; n \in \mathbb{N}_{0}\right),
\end{gathered}
$$

on condition that the integral in (1.11) exists.

Now, on using the definition (1.10), (1.11) can be written as

$$
\begin{gathered}
\int_{-1}^{1}(1-z)^{\xi-1}(1+z)^{\eta-1} P_{n}^{(\alpha, \beta)}(z) d z= \\
2^{\xi+\eta-1}\left(\begin{array}{c}
\alpha+n \\
n
\end{array}\right) B(\xi, \eta)_{3} F_{2}\left[\begin{array}{c}
-n, \alpha+\beta+n+1, \xi ; \\
\alpha+1, \xi+\eta ;
\end{array}\right] \\
(\min \{\Re(\xi), \Re(\eta)\}>0),
\end{gathered}
$$

Lately, Srivastava et al. [17] introduced an interesting Jacobi transform formula

$$
\begin{aligned}
\rrbracket^{\alpha, \beta}\left[z^{\rho-1} ; n\right] & =\int_{-1}^{1}(1-z)^{\alpha}(1+z)^{\beta} P_{n}^{(\alpha, \beta)}(z) z^{\rho-1} d z \\
& =2^{\alpha+\beta+1}\left(\begin{array}{c}
\alpha+n \\
n
\end{array}\right) B(\alpha+1, \beta+1)
\end{aligned}
$$




$$
\begin{gathered}
. F_{1: 1: 0}^{1: 2 ; 1}\left[\begin{array}{rr}
\alpha+1:-n \alpha+\beta+n+1 ; 1-\rho ; \\
\alpha+\beta+2: \quad \alpha+1 ;---;
\end{array}\right] \\
\left(\min \{\Re(\alpha), \Re(\beta)\}>-1 ; \rho \in \mathbb{C} ; n \in \mathbb{N}_{0}\right),
\end{gathered}
$$

in terms of the Kampé de Fériet function (see, for details, [9, p.27 et. seq.]).

It holds curiosity that the Jacobi polynomials $P_{n}^{(\alpha, \beta)}(z)$ as their special case, becomes other polynomials being the Legendre polynomials $P_{n}(z)$, the Chebyshev polynomials $T_{n}(z)$ and $U_{n}(z)$ (first and second kind), the Gegenbauer polynomials $C_{n}^{v}(z)$. We have indeed

$$
C_{n}^{v}(z)=\left(\begin{array}{c}
v+n-\frac{1}{2} \\
n
\end{array}\right)^{-1}\left(\begin{array}{c}
2 v+n-1 \\
n
\end{array}\right) P_{n}^{v-\frac{1}{2}, v-\frac{1}{2}}(z)
$$

and

$$
P_{n}(z)=C_{n}^{\frac{1}{2}}(z)=P_{n}^{(0,0)}(z),
$$

respectively. In view of above relationships, the Gegenbauer transform $\mathbb{G}^{v}[f(z) ; n]$ and the Legendre transform $\mathbb{L}[f(z) ; n]$ (see [17]) can be defined as:

$$
\begin{aligned}
\mathbb{G}^{v}[f(z) ; n] & =\left(\begin{array}{c}
v+n-\frac{1}{2} \\
n
\end{array}\right)^{-1}\left(\begin{array}{c}
2 v+n-1 \\
n
\end{array}\right) \mathbb{J}^{v-\frac{1}{2}, v-\frac{1}{2}}[f(z) ; n] \\
& :=\int_{-1}^{1}\left(1-z^{2}\right)^{v-\frac{1}{2}} C_{n}^{(v)}(z) f(z) d z \quad\left(\Re(v)>-\frac{1}{2} ; n \in \mathbb{N}_{0}\right)
\end{aligned}
$$

and

$$
\mathbb{L}[f(z) ; n]=\mathbb{G}^{\frac{1}{2}}[f(z) ; n]=\int_{-1}^{1} P_{n}(z) f(z) d z \quad\left(n \in \mathbb{N}_{0}\right) .
$$

Definition 1.1. Using the definition in (1.9), we now define a new generalization of the Wright function as follows:

$$
\begin{aligned}
& W_{\alpha, \beta, \gamma, \delta, \lambda, \sigma}^{\left(\left\{v_{m}\right\}_{m \in N_{0}}\right)}(z ; p)=\sum_{n=0}^{\infty} \frac{B_{p}^{\left(\left\{v_{m}\right\}_{m \in \mathbb{N}_{0}}\right)}(\gamma+n, \lambda-\gamma)}{B(\gamma, \lambda-\gamma)} \frac{z^{n}}{n ! \Gamma(\alpha n+\beta)} \frac{(\sigma)_{n}}{(\delta)_{n}}, \\
& (\alpha>-1, \delta \neq 0,-1,-2, \ldots ; \gamma, \delta, \lambda, \sigma, \beta, \alpha \in \mathbb{C} \text { with } z \in \mathbb{C} \text { and }|z|<1) .
\end{aligned}
$$

We also introduce the two Wright type auxillary functions, respectively as

$$
M_{\alpha, \gamma, \delta, \lambda, \sigma}^{\left(\left\{v_{m}\right\}_{m \in \mathbb{N}_{0}}\right)}(z ; p)=W_{-\alpha, 1-\alpha, \gamma, \delta, \lambda, \sigma}^{\left(\left\{v_{m}\right\}_{m \in \mathbb{N}_{0}}\right)}(-z ; p)=\sum_{n=0}^{\infty} \frac{(\sigma)_{n}}{(\delta)_{n}} \frac{(-1)^{n} z^{n}}{n !} \frac{(\gamma)_{n}}{\Gamma(1-\alpha n+\alpha)(\lambda)_{n}}
$$

and

$$
F_{\alpha, \gamma, \delta, \lambda, \sigma}^{\left(\left\{v_{m}\right\}_{m \in \mathbb{N}_{0}}\right)}(z ; p)=W_{\alpha, 0, \gamma, \delta, \lambda, \sigma}^{\left(\left\{v_{m}\right\}_{m \in \mathbb{N}_{0}}\right)}(-z ; p)=\sum_{n=0}^{\infty} \frac{(\sigma)_{n}}{(\delta)_{n}} \frac{(-1)^{n} z^{n}}{n !} \frac{(\gamma)_{n}}{(\lambda)_{n} \Gamma(-\alpha n)} .
$$


In the subsequent sections, we study certain properties and formulas involving the new generalized Wright function. We derive certain results involving Jacobi, Gegenbauer, Legendre and Mellin transform. Also, using an extended Riemann-Liouville fractional derivative, we present a fractional derivative formula (3.13) for the generalized Wright function. Many authors have also built up integrals and other formulas including a variety of special functions (see, for more work, $[1,10,12,13]$ ). Many interesting properties in terms of derivative and integral formulas concerning Wright function have been established. Recently, a noticeable effort of Shahid and Salem [5] can be seen in presenting the new extension of Wright function.

\section{Integral formulas involving generalized Wright function}

Theorem 2.1. Let $\alpha>-1, c, \lambda, \beta, \gamma, \delta, \sigma \in \mathbb{C}$ with $\Re(\delta)>\Re(\gamma)>0$. Then

$$
W_{\alpha, \beta, \gamma, \delta, \lambda, \sigma}^{\left(\left\{v_{m}\right\}_{m \in \mathbb{N}_{0}}\right)}(z ; p)=\frac{1}{B(\gamma, \lambda-\gamma)} \int_{0}^{1}(1-t)^{\lambda-\gamma-1} t^{\gamma-1} \mathscr{F}\left[\left\{v_{m}\right\}_{m \in \mathbb{N}_{0}} ;-\frac{p}{(1-t) t}\right] W_{\alpha, \beta}^{\sigma, \delta}(t z) d t .
$$

Proof. Using (1.9) in (1.18), we have

$$
\begin{aligned}
W_{\alpha, \beta, \gamma, \delta, \lambda, \sigma}^{\left(\left\{v_{m}\right\}_{m \in \mathbb{N}_{0}}\right)}(z ; p)= & \sum_{n=0}^{\infty}\left\{\int_{0}^{1}(1-t)^{\lambda-\gamma-1} t^{\gamma+n-1} \mathscr{F}\left[\left\{v_{m}\right\}_{m \in \mathbb{N}_{0}} ;-\frac{p}{(1-t) t}\right] d t\right\} \\
& \times \frac{(\sigma)_{n}}{(\delta)_{n} B(\gamma, \lambda-\gamma)} \frac{z^{n}}{\Gamma(\alpha n+\beta) n !} .
\end{aligned}
$$

On interchanging the order of integration and summation in the equation above, which is proven under the given conditions here, we get

$$
W_{\alpha, \beta, \gamma, \delta, \lambda, \sigma}^{\left(\left\{v_{m}\right\}_{m \in \mathbb{N}_{0}}\right)}(z ; p)=\int_{0}^{1} t^{\gamma-1}(1-t)^{\lambda-\gamma-1} \mathscr{F}\left[\left\{v_{m}\right\}_{m \in \mathbb{N}_{0}} ; \frac{-p}{(1-t) t}\right] \sum_{n=0}^{\infty} \frac{(t z)^{n}}{n ! \Gamma(\alpha n+\beta)} \frac{(\sigma)_{n}}{(\delta)_{n} B(\gamma, \lambda-\gamma)} d t .
$$

Using (1.4) in the precedent equation, we get the desired result, and in view of (1.19) and (1.20), one can consequently get

$$
\begin{aligned}
M_{\alpha, \gamma, \delta, \lambda, \sigma}^{\left(\left\{v_{m}\right\}_{m \in \mathbb{N}_{0}}\right)}(z ; p)= & \frac{1}{B(\gamma, \lambda-\gamma)} \\
& \times \int_{0}^{1}(1-t)^{\lambda-\gamma-1} t^{\gamma-1} \mathscr{F}\left[\left\{v_{m}\right\}_{m \in \mathbb{N}_{0}} ;-\frac{p}{(1-t) t}\right] M_{\alpha}^{\sigma, \delta}(-t z) d t
\end{aligned}
$$

and

$$
\begin{aligned}
F_{\alpha, \gamma, \delta, \lambda, \sigma}^{\left(\left\{v_{m}\right\}_{m \in \mathbb{N}_{0}}\right)}(z ; p)= & \frac{1}{B(\gamma, \lambda-\gamma)} \\
& \times \int_{0}^{1}(1-t)^{\lambda-\gamma-1} t^{\gamma-1} \mathscr{F}\left[\left\{v_{m}\right\}_{m \in \mathbb{N}_{0}} ;-\frac{p}{(1-t) t}\right] F_{\alpha}^{\sigma, \delta}(-t z) d t .
\end{aligned}
$$

Now, letting $t=\frac{u}{1+u}$ and $t=\sin ^{2} \theta$ in (2.1), we come up with two interesting integral formulas for the extended Wright function in (1.18), which are given in the respective Corollaries. 
Corollary 2.2. Let $c, \gamma, \beta, \alpha, \delta, \lambda, \sigma \in \mathbb{C}$ with $\Re(\delta)>\Re(\gamma)>0$ and $\Re(\alpha)>0$. Also let $p \in \mathbb{C}$ and $d \in \mathbb{C} \backslash Z_{o}^{-}$. Then

$$
W_{\alpha, \beta, \gamma, \delta, \lambda, \sigma}^{\left(\left\{v_{m}\right\}_{m \in \mathbb{N}_{0}}\right)}(z ; p)=\frac{1}{B(\gamma, \lambda-\gamma)} \int_{0}^{\infty} \frac{u^{\gamma-1}}{(1+u)^{\lambda}} \mathscr{F}\left[\left\{v_{m}\right\}_{m \in \mathbb{N}_{0}} ;-\frac{p(1+u)^{2}}{u}\right] W_{\alpha, \beta}^{\sigma, \delta}\left(\frac{u z}{1+u}\right) d u
$$

Corollary 2.3. Let $\gamma, \alpha, \beta, \delta, \lambda, \sigma, \in \mathbb{C}$ with $\Re(\delta)>\Re(\gamma)>0$ and $\Re(\alpha)>0$.

Also, let $p \in \mathbb{R}_{0}^{+}$. Then

$$
\begin{aligned}
& W_{\alpha, \beta, \gamma, \delta, \lambda, \sigma}^{\left(\left\{v_{m}\right\}_{m \in N_{0}}\right)}(z ; p)=\frac{2}{B(\gamma, \lambda-\gamma)} \\
& \quad \times \int_{0}^{\frac{\pi}{2}} \sin ^{2 \gamma-1} \theta \cos ^{2 \lambda-2 \gamma-1} \theta \mathscr{F}\left[\left\{v_{m}\right\}_{m \in \mathbb{N}_{0}} ;-\frac{p}{\sin ^{2} \theta \cos ^{2} \theta}\right] W_{\alpha, \beta}^{\sigma, \delta}\left(z \sin ^{2} \theta\right) d \theta .
\end{aligned}
$$

Theorem 2.4. The Mellin transform of the generalized Wright function is given by

$$
\begin{aligned}
& \mathfrak{M}\left\{W_{\alpha, \beta, \gamma, \delta, \lambda, \sigma}^{\left(\left\{v_{m}\right\}_{m \in N_{0}}\right)}(z ; p) ; s\right\}=\int_{0}^{\infty} p^{s-1} W_{\alpha, \beta, \gamma, \delta, \lambda, \sigma}^{\left(\left\{v_{m}\right\}_{m \in \mathbb{N}_{0}}\right)}(z ; p) d p \\
& =\frac{\Gamma_{p}^{\left(\left\{v_{m}\right\}_{m \in \mathbb{N}_{0}}\right)}(s) \Gamma(\lambda+s-\gamma) \Gamma(\delta) \Gamma(\lambda)}{\Gamma(\gamma) \Gamma(\sigma) \Gamma(\lambda-\gamma)}{ }_{2} \psi_{3}\left[\begin{array}{ll}
(\sigma, 1),(\gamma+s, 1) ; & \\
(\delta, 1), \quad(\beta, \alpha), & (\lambda+2 s, 1) ;
\end{array}\right] .
\end{aligned}
$$

Proof. Appplying (2.1) to (2.6), we have

$$
\begin{aligned}
& \mathfrak{M}\left\{W_{\alpha, \beta, \gamma, \delta, \lambda, \sigma}^{\left(\left\{v_{m}\right\}_{m \in N_{0}}\right)}(z ; p) ; s\right\}=\frac{1}{B(\gamma, \lambda-\gamma)} \\
& \quad \times \int_{0}^{\infty} p^{s-1} \times \int_{0}^{1} t^{\gamma-1}(1-t)^{\lambda-\gamma-1} \mathscr{F}\left[\left\{v_{m}\right\}_{m \in \mathbb{N}_{0}} ;-\frac{p}{(1-t) t}\right] W_{\alpha, \beta}^{\sigma, \delta}(t z) d t d p .
\end{aligned}
$$

On interchanging the order of integration in (2.7), guarenteed under the conditions here, we get

$$
\begin{aligned}
& \mathfrak{M}\left\{W_{\alpha, \beta, \gamma, \delta, \lambda, \sigma}^{\left(\left\{v_{m}\right\}_{m \in \mathbb{N}_{0}}\right)}(z ; p) ; s\right\}=\frac{1}{B(\gamma, \lambda-\gamma)} \\
& \quad \times \int_{0}^{1}\left\{t^{\gamma-1}(1-t)^{\lambda-\gamma-1} W_{\alpha, \beta}^{\sigma, \delta}(t z)\right\} \times \int_{0}^{\infty} p^{s-1} \mathscr{F}\left[\left\{v_{m}\right\}_{m \in \mathbb{N}_{0}} ;-\frac{p}{(1-t) t}\right] d p d t .
\end{aligned}
$$

Substituting $u=\frac{p}{t(1-t)}$ in the inner integral in (2.8), we get

$$
\begin{aligned}
\int_{0}^{\infty} p^{s-1} \mathscr{F}\left[\left\{v_{m}\right\}_{m \in \mathbb{N}_{0}} ;-u\right] d p & =\int_{0}^{\infty} u^{s-1} t^{s}(1-t)^{s} \mathscr{F}\left[\left\{v_{m}\right\}_{m \in \mathbb{N}_{0}} ;-\frac{p}{(1-t) t}\right] d u \\
& =t^{s}(1-t)^{s} \int_{0}^{\infty} u^{s-1} \mathscr{F}\left[\left\{v_{m}\right\}_{m \in \mathbb{N}_{0}} ;-u\right] d u \\
& =t^{s}(1-t)^{s} \Gamma_{p}^{\left(\left\{v_{m}\right\}_{m \in N_{0}}\right)}(s)
\end{aligned}
$$


where $\Gamma_{p}^{\left(\left\{v_{m}\right\}_{m \in \mathbb{N}_{0}}\right)}(s)$ is the extended gamma function [17] defined as

$$
\Gamma_{p}^{\left(\left\{v_{m}\right\}_{m \in \mathbb{N}_{0}}\right)}(s)=\int_{0}^{\infty} t^{s-1} \mathscr{F}\left[\left\{v_{m}\right\}_{m \in \mathbb{N}_{0}} ;-t-\frac{p}{t}\right] d t .
$$

Using (2.10) and (2.8), we find

$$
\begin{aligned}
\mathfrak{M}\left\{W_{\alpha, \beta, \gamma, \delta, \lambda, \sigma}^{\left(\left\{v_{m}\right\}_{m \in \mathbb{N}_{0}}\right)}(z ; p) ; s\right\}=\frac{\Gamma_{p}^{\left(\left\{v_{m}\right\}_{m \in \mathbb{N}_{0}}\right)}(s)}{B(\gamma, \lambda-\gamma)} \int_{0}^{1} t^{\gamma+n+s-1}(1-t)^{\lambda+s-\gamma-1} W_{\alpha, \beta}^{\sigma, \delta}(t z) d t \\
=\frac{\Gamma_{p}^{\left(\left\{v_{m}\right\}_{m \in \mathbb{N}_{0}}\right)}(s)}{B(\gamma, \lambda-\gamma)} \sum_{n=0}^{\infty} \frac{(\sigma)_{n}}{(\delta)_{n}} \frac{z^{n}}{n ! \Gamma(\alpha n+\beta)} \int_{0}^{1} t^{\gamma+n+s-1}(1-t)^{\lambda+s-\gamma-1} d t \\
=\frac{\Gamma_{p}^{\left(\left\{v_{m}\right\}_{m \in \mathbb{N}_{0}}\right)}(s)}{B(\gamma, \lambda-\gamma)} \sum_{n=0}^{\infty} \frac{(\sigma)_{n}}{(\delta)_{n}} \frac{z^{n}}{n ! \Gamma(\alpha n+\beta)} \times \frac{\Gamma(\lambda+s-\gamma) \Gamma(\gamma+n+s)}{\Gamma(\lambda+n+2 s)} \\
=\frac{\Gamma(\lambda+s-\gamma) \Gamma_{p}^{\left(\left\{v_{m}\right\}_{m \in \mathbb{N}_{0}}\right)}(s) \Gamma(\lambda)}{\Gamma(\lambda-\gamma) \Gamma(\gamma)} \sum_{n=0}^{\infty} \frac{\Gamma(\sigma+n) \Gamma(\gamma+n+s) \Gamma(\delta)}{\Gamma(\lambda+n+2 s) \Gamma(\alpha n+\beta) \Gamma(\delta+n) \Gamma(\sigma)} \frac{z^{n}}{n !} \\
=\frac{\Gamma(\lambda+s-\gamma) \Gamma_{p}^{\left(\left\{v_{m}\right\}_{m \in \mathbb{N}_{0}}\right)}(s)}{\Gamma(\lambda-\gamma)} \frac{\Gamma(\delta) \Gamma(\lambda)}{\Gamma(\gamma) \Gamma(\sigma)} \sum_{n=0}^{\infty} \frac{\Gamma(\sigma+n)}{\Gamma(\alpha n+\beta) \Gamma(\delta+n)} \frac{\Gamma(\gamma+n+s)}{\Gamma(\lambda+n+2 s)} \frac{z^{n}}{n !},
\end{aligned}
$$

which in terms of Fox-Wright function $\psi_{q}$, yields

$$
\mathfrak{M}\left\{W_{\alpha, \beta, \gamma, \delta, \lambda, \sigma}^{\left(\left\{v_{m}\right\}_{m \in N_{0}}\right)}(z ; p) ; s\right\}=\frac{\Gamma_{p}^{\left(\left\{v_{m}\right\}_{m \in \mathbb{N}_{0}}\right)}(s) \Gamma(\lambda+s-\gamma) \Gamma(\delta) \Gamma(\lambda)}{\Gamma(\gamma) \Gamma(\sigma) \Gamma(\lambda-\gamma)}{ }_{2} \psi_{3}\left[\begin{array}{ll}
(\sigma, 1),(\gamma+s, 1) ; & z \\
(\delta, 1), \quad(\beta, \alpha), \quad(\lambda+2 s, 1) ;
\end{array}\right] .
$$

Corollary 2.5. Taking $s=1$ in Theorem 2.4 and using $\Gamma^{(c, d)}(1)=\frac{\Gamma(d) \Gamma(c-1)}{\Gamma(c) \Gamma(d-1)}$, we get

$$
\mathfrak{M}\left\{W_{\alpha, \beta, \gamma, \delta, \lambda, \sigma}^{\left(\left\{v_{m}\right\}_{m \in N_{0}}\right)}(z ; p) ; 1\right\}=\frac{\Gamma(\delta) \Gamma(\lambda)}{\Gamma(\gamma) \Gamma(\sigma)} \frac{\Gamma(c-1) \Gamma(d) \Gamma(\lambda+1-\gamma)}{\Gamma(c) \Gamma(d-1) \Gamma(\lambda-\gamma)}{ }_{2} \psi_{3}\left[\begin{array}{ll}
(\sigma, 1),(\gamma+1,1) ; & z \\
(\delta, 1), \quad(\beta, \alpha), \quad(\lambda+2,1) ;
\end{array}\right] .
$$

Corollary 2.6. By the Mellin inversion formula, we have the following complex integral representation for $W_{\alpha, \beta, \gamma, \delta, \lambda, \sigma}^{\left(\left\{v_{m}\right\}_{m \in N_{0}}\right)}(z ; p)$ :

$$
\begin{aligned}
W_{\alpha, \beta, \gamma, \delta, \lambda, \sigma}^{\left(\left\{v_{m}\right\}_{m \in \mathbb{N}_{0}}\right)}(z ; p)= & \frac{1}{2 \pi i} \int_{-i \infty}^{i \infty} \frac{\Gamma_{p}^{\left(\left\{v_{m}\right\}_{m \in \mathbb{N}_{0}}\right)}(s) \Gamma(\lambda+s-\gamma) \Gamma(\delta) \Gamma(\lambda)}{\Gamma(\gamma) \Gamma(\sigma) \Gamma(\lambda-\gamma)} \\
& \times{ }_{2} \psi_{3}\left[\begin{array}{lr}
(\sigma, 1),(\gamma+s, 1) ; \\
(\delta, 1), \quad(\beta, \alpha), \quad(\lambda+2 s, 1) ;
\end{array}\right] p^{-s} d s .
\end{aligned}
$$


We present a recurrence relation for (1.18) given by the undermentioned corollary:

Corollary 2.7. Let $\gamma, \delta, \lambda, \sigma, \alpha, \beta \in \mathbb{C}$ with $\Re(\delta)>\Re(\gamma)>0$ and $\Re(\alpha)>0$. Also, let $p \in \mathbb{R}_{0}^{+}$. Then

$$
W_{\alpha, \beta, \gamma, \delta, \lambda, \sigma}^{\left(\left\{v_{m}\right\}_{m \in \mathbb{N}_{0}}\right)}(z ; p)=\beta W_{\alpha, \beta+1, \gamma, \delta, \lambda, \sigma}^{\left(\left\{v_{m}\right\}_{m \in \mathbb{N}_{0}}\right)}(z ; p)+\frac{\alpha \sigma \gamma z}{\lambda \delta} W_{\alpha, \alpha+\beta+1, \gamma+1, \delta+1, \lambda+1, \sigma+1}^{\left(\left\{v_{m}\right\}_{m \in \mathbb{N}_{0}}\right)}(z ; p) .
$$

Proof. By using (3.12), it is easy to obtain the desired result. The detailed proof is omitted.

Theorem 2.8. With the conditions already mentioned for (1.18), the following Jacobi transform is true:

$$
\begin{aligned}
& \mathbb{J}^{\alpha, \beta}\left[z^{\rho-1} W_{\alpha, \beta, \gamma, \delta, \lambda, \sigma}^{\left(\left\{v_{m}\right\}_{m \in \mathbb{N}_{0}}\right)}(x z ; p) ; n\right]= 2^{\alpha+\beta+1}\left(\begin{array}{c}
\alpha+n \\
n
\end{array}\right) B(\alpha+1, \beta+1) \sum_{k=0}^{\infty} \mho_{k} \\
& . F_{1: 1: 0}^{1: 2 ; 1}\left[\begin{array}{c}
\alpha+1:-n, \alpha+\beta+n+1 ; 1-\rho-k ; \\
\alpha+\beta+2: \quad \alpha+1 ;---;
\end{array}\right] \frac{x^{k}}{k !} \\
&\left(|x|<1 ; n \in \mathbb{N}_{0} ; \min \{\Re(\alpha), \Re(\beta)\}>-1 ; \rho \in \mathbb{C}\right),
\end{aligned}
$$

where, the coefficients $\mho_{k}$ are given by

$$
\mho_{k}=\sum_{k=0}^{\infty} \frac{B_{p}^{\left(\left\{v_{m}\right\}_{m \in N_{0}}\right)}(\gamma+k, \lambda-\gamma)}{B(\gamma, \lambda-\gamma)} \frac{(\sigma)_{k}}{\Gamma(\alpha k+\beta)(\delta)_{k}},
$$

and we assume that the Jacobi transform in (2.15) exists.

Proof. Applying the defintion (1.11) in concurrence with (1.18), we have

$$
\begin{aligned}
\rrbracket^{\alpha, \beta}\left[z^{\rho-1} W_{\alpha, \beta, \gamma, \delta, \lambda, \sigma}^{\left(\left\{v_{m}\right\}_{m \in \mathbb{N}_{0}}\right)}(x z ; p) ; n\right] & =\int_{-1}^{1} z^{\rho-1}(1+z)^{\beta}(1-z)^{\alpha} P_{n}^{\alpha, \beta}(z) . W_{\alpha, \beta, \gamma, \delta, \lambda, \sigma}^{\left(\left\{v_{m}\right\}_{m \in N_{0}}\right)}(x z ; p) d z \\
& =\int_{-1}^{1} z^{\rho-1}(1+z)^{\beta}(1-z)^{\alpha} P_{n}^{\alpha, \beta}(z)\left(\sum_{k=0}^{\infty} \mho_{k} \frac{(x z)^{k}}{k !}\right) d z,
\end{aligned}
$$

where $\mho_{k}$ are given by (2.16). Now, by changing the summation and integration order, and applying the Jacobi transform formula (1.13) with $\rho$ replaced by $\rho+k$, we get the required statement (2.15) under the hypotheses of Theorem 2.8 .

Theorem 2.9. With the conditions mentioned in (1.18), the following Gegenbauer transform holds:

$$
\begin{aligned}
& \mathbb{G}^{v}\left[z^{\rho-1} W_{\alpha, \beta, \gamma, \delta, \lambda, \sigma}^{\left(\left\{v_{m}\right\}_{m \in \mathbb{N}_{0}}\right)}(x z ; p) ; n\right]= 2^{2 v}\left(\begin{array}{c}
2 v+n-1) \\
n
\end{array}\right) B\left(v+\frac{1}{2}, v+\frac{1}{2}\right) \sum_{k=0}^{\infty} \mho_{k} \\
& . F_{1: 1: 0}^{1: 2 ; 1}\left[\begin{array}{cc}
v+\frac{1}{2}: & -n 2 v+n ; 1-\rho-k ; \\
2 v+1: & v+\frac{1}{2} ; \quad---; 1,2
\end{array}\right] \frac{x^{k}}{k !} \\
&\left(|x|<1 ; n \in \mathbb{N}_{0} ; \Re(v)>-\frac{1}{2} ; \rho \in \mathbb{C}\right),
\end{aligned}
$$

provided that the Gegenbauer transform in (2.18) exists. 
Remark 2.10. Note that the above transform is precisely the particular case of Theorem 2.8 for $\alpha=\beta=v-\frac{1}{2}$.

For $\alpha=\beta=0$ in Theorem 2.8, we get the Legendre transform as a particular case of Theorem 2.8 given under:

Theorem 2.11. With the conditions mentioned with (1.18), the following Legendre transform formula holds true:

$$
\begin{aligned}
& \mathbb{L}\left[z^{\rho-1} W_{\alpha, \beta, \gamma, \delta, \lambda, \sigma}^{\left(\left\{v_{m}\right\}_{m \in \mathbb{N}_{0}}\right)}(x z ; p) ; n\right]=2 \sum_{k=0}^{\infty} \mho_{k} \cdot F_{1: 1: 0}^{1: 2 ; 1}\left[\begin{array}{cc}
1: & -n, n+1 ; 1-\rho-k ; 1,2 \\
2: & 1 ;
\end{array}\right] \frac{x^{k}}{k !} \\
& \left(|x|<1 ; n \in \mathbb{N}_{0} ; \rho \in \mathbb{C}\right),
\end{aligned}
$$

provided that the Legendre transform in (2.19) exists and the coefficients $\mho_{k}$ are given by (2.16).

\section{Derivative Formulas involving generalized Wright function}

We recall the Riemann-Liouville fractional derivative of order $(\mu \in \mathbb{C} ; \Re(\mu) \geq 0)$ defined by

$$
\begin{aligned}
\mathfrak{D}_{x}^{\mu}\{f(x)\} & =\frac{1}{\Gamma(r-\mu)} \frac{d^{r}}{d x^{r}} \int_{0}^{x} f(t)(x-t)^{-\mu+r-1} d t \\
(r & \in \mathbb{N} ; r-1<\Re(\mu)<r ; x>0) .
\end{aligned}
$$

In particular,

$$
\begin{aligned}
\mathfrak{D}_{x}^{\mu}\{f(x)\}= & \frac{1}{\Gamma(1-\mu)} \frac{d}{d x} \int_{0}^{x} f(t)(x-t)^{-\mu} d t \\
& (0<\Re(\mu)<1 ; x>0) .
\end{aligned}
$$

A more generalized form of (3.1) introduced by Agarwal et al. [3] is defined as

$$
\begin{gathered}
\mathfrak{D}_{x ; c, d}^{\mu, p}\{f(x)\}=\frac{1}{\Gamma(r-\mu)} \frac{d^{r}}{d x^{r}} \int_{0}^{x} f(t)(x-t)^{-\mu+r-1}{ }_{1} F_{1}\left[c, d ; \frac{-p x^{2}}{t(x-t)}\right] d t \\
\left(r \in \mathbb{N} ; r>\Re(\mu)>r-1 ; x \in \mathbb{R}^{+} ; p \in \mathbb{R}_{0}^{+} ; c \in \mathbb{C} ; d \in \mathbb{C} \backslash \mathbb{Z}_{0}^{+}\right) .
\end{gathered}
$$

In particular,

$$
\begin{gathered}
\mathfrak{D}_{x ; c, d}^{\mu, p}\{f(x)\}=\frac{1}{\Gamma(1-\mu)} \frac{d}{d x} \int_{0}^{x}(x-t)^{-\mu} f(t)_{1} F_{1}\left[c, d ; \frac{-p x^{2}}{t(x-t)}\right] d t \\
\left(p \in \mathbb{R}_{0}^{+} ; 0<\Re(\mu)<1 ; x \in \mathbb{R}^{+} ; c \in \mathbb{C}, d \in \mathbb{C} \backslash \mathbb{Z}_{0}^{+}\right) .
\end{gathered}
$$

The special case of (3.1) when and $p=0$ becomes the classical Reimann-Liouville fractional derivative. 
The generalization of the extended Reimann-Liouville fractional derivative of a function $f(x)$ of order $\mu$ [19] is defined by

$$
\begin{gathered}
\mathfrak{D}_{x ; \mu, p}^{\left(\left\{v_{m}\right\}_{m \in \mathbb{N}_{0}}\right)}\{f(x)\}=\frac{1}{\Gamma(-\mu)} \int_{0}^{x} f(t)(x-t)^{-\mu-1} \mathscr{F}\left[\left\{v_{m}\right\}_{m \in \mathbb{N}_{0}} ; \frac{-p x^{2}}{t(x-t)}\right] d t \\
\left(\Re(p)>0 ; \Re(\mu)<0 ; x \in \mathbb{R}^{+}\right) .
\end{gathered}
$$

When $\Re(\mu) \geq 0$, let $r \in \mathbb{N}$ be the smallest integer greater than $\Re(\mu)$ and so $r-1 \leq \Re(\mu)<r$. Then the generalized Riemann-Liouville fractional derivative of $f(x)$ of order $\mu$ is defined by

$$
\begin{aligned}
& \mathfrak{D}_{x ; \mu, p}^{\left(\left\{v_{m}\right\}_{m \in \mathbb{N}_{0}}\right)}:=\frac{d^{r}}{d x^{r}} \mathfrak{D}_{x ; \mu-r, p}^{\left(\left\{v_{m}\right\}_{m \in N_{0}}\right)}\{f(x)\} \\
& =\frac{d^{r}}{d x^{r}}\left\{\frac{1}{\Gamma(r-\mu)} \int_{0}^{x} f(t)(x-t)^{-\mu+r-1} \mathscr{F}\left[\left\{v_{m}\right\}_{m \in \mathbb{N}_{0}} ; \frac{-p x^{2}}{t(x-t)}\right] d t\right\} \\
& \left(\Re(\mu)>0 ; x \in \mathbb{R}^{+} ; p \in \mathbb{R}_{0}^{+}\right) .
\end{aligned}
$$

We first apply this fractional derivative on a function $z^{\tau}$ under the following Lemma:

Lemma 3.1. Let $r-1 \leq \Re(\mu)<r$ for some $r \in \mathbb{N}$ and $\Re(\mu)<\Re(\tau)$. Then we have

$$
\mathfrak{D}_{x ; \mu, p}^{\left(\left\{v_{m}\right\}_{m \in \mathbb{N}_{0}}\right)}\left\{z^{\tau}\right\}=\frac{\Gamma(\tau+1) B_{p}^{\left(\left\{v_{m}\right\}_{m \in \mathbb{N}_{0}}\right)}(\tau+1, r-\mu)}{\Gamma(\tau-\mu+1) B(\tau+1, r-\mu)} z^{\tau-\mu} .
$$

Proof. Applying (3.6) to the function $z^{\tau}$, we have

$$
\mathfrak{D}_{z ; \mu, p}^{\left(\left\{v_{m}\right\}_{m \in \mathbb{N}_{0}}\right)}\left\{z^{\tau}\right\}=\frac{d^{r}}{d z^{r}}\left\{\frac{1}{\Gamma(r-\mu)} \int_{0}^{z} t^{\tau}(z-t)^{-\mu+r-1} \mathscr{F}\left[\left\{v_{m}\right\}_{m \in \mathbb{N}_{0}} ; \frac{-p z^{2}}{t(z-t)}\right] d t\right\} .
$$

Setting $t=z u$ above, we get

$$
\mathfrak{D}_{z ; \mu, p}^{\left(\left\{v_{m}\right\}_{m \in \mathbb{N}_{0}}\right)}\left\{z^{\tau}\right\}=\left(\frac{d^{r}}{d z^{r}} z^{r+\tau-\mu}\right) \times \frac{1}{\Gamma(r-\mu)} \int_{0}^{z} u^{\tau+1-1}(1-u)^{-\mu+r-1} \mathscr{F}\left[\left\{v_{m}\right\}_{m \in \mathbb{N}_{0}} ; \frac{-p}{u(1-u)}\right] d u .
$$

Now, since

$$
\frac{d^{r}}{d z^{r}} z^{r+\tau-\mu}=\frac{\Gamma(1+\tau-\mu+r)}{\Gamma(1+\tau-\mu)} z^{\tau-\mu}
$$

therefore, in view of (1.9), we get the desired result.

Lemma 3.2. The following easily derivable formulas for the usual beta function $B(x, y)$ and $B_{p}^{c, d}(x, y)$ in (1.8) are mentioned in the lemma below:

$$
\begin{gathered}
B(u, v+1)=\frac{v}{u+v} B(u, v), \\
B(u+1, v)=\frac{u}{u+v} B(u, v), \\
B_{p}^{\left(\left\{v_{m}\right\}_{m \in N_{0}}\right)}(u, v)=B_{p}^{\left(\left\{v_{m}\right\}_{m \in N_{0}}\right)}(u+1, v)+B_{p}^{\left(\left\{v_{m}\right\}_{m \in N_{0}}\right)}(u, v+1) .
\end{gathered}
$$


Now, we present a derivative formula for the generalized Wright function (1.18), asserted by the following theorem:

Theorem 3.3. Let $\Re(\alpha)>0, p \in \mathbb{R}_{0}^{+}, \Re(\delta)>\Re(\gamma)>0$ and $d \in \mathbb{C} \backslash \mathbb{Z}_{o}^{-}$. Then

$$
\frac{d^{n}}{d z^{n}} W_{\alpha, \beta, \gamma, \delta, \lambda, \sigma}^{\left(\left\{v_{m}\right\}_{m \in N_{0}}\right)}(z ; p)=\frac{(\gamma)_{n}(\sigma)_{n}}{(\delta)_{n}(\lambda)_{n}} W_{\alpha, n \alpha+\beta, \gamma+n, \delta+n, \lambda+n, \sigma+n}^{\left(\left\{v_{m}\right\}_{m \in N_{0}}\right)}(z ; p) .
$$

Proof. From (1.18), we have

$$
\begin{aligned}
\frac{d}{d z} W_{\alpha, \beta, \gamma, \delta, \lambda, \sigma}^{\left(\left\{v_{m}\right\}_{m \in N_{0}}\right)}(z ; p) & =\sum_{n=1}^{\infty} \frac{B_{p}^{\left(\left\{v_{m}\right\}_{m \in \mathbb{N}_{0}}\right)}(\gamma+n, \lambda-\gamma)}{B(\gamma, \lambda-\gamma)} \frac{(z)^{n-1}}{(n-1) ! \Gamma(\alpha n+\beta)} \frac{(\sigma)_{n}}{(\delta)_{n}} \\
& =\sum_{n=0}^{\infty} \frac{(\sigma)_{n+1}}{(\delta)_{n+1}} \frac{B_{p}^{\left(\left\{v_{m}\right\}_{m \in N_{0}}\right)}(\gamma+n+1, \lambda-\gamma)}{B(\gamma, \lambda-\gamma)} \frac{(z)^{n}}{n ! \Gamma(\alpha n+\alpha+\beta)} \\
& =\frac{\sigma}{\delta} \sum_{n=0}^{\infty} \frac{(\sigma+1)_{n}}{(\delta+1)_{n}} \frac{B_{p}^{\left(\left\{v_{m}\right\}_{m \in N_{0}}\right)}(\gamma+n+1, \lambda-\gamma)}{B(\gamma, \lambda-\gamma)} \frac{z^{n}}{n ! \Gamma(\alpha n+\alpha+\beta)}
\end{aligned}
$$

Now, using (3.9), we get

$$
\frac{d}{d z} W_{\alpha, \beta, \gamma, \delta, \lambda, \sigma}^{\left(\left\{v_{m}\right\}_{m \in \mathbb{N}_{0}}\right)}(z ; p)=\frac{\sigma \gamma}{\delta \lambda} \sum_{n=0}^{\infty} \frac{(\sigma+1)_{n}}{(\delta+1)_{n}} \frac{B_{p}^{\left(\left\{v_{m}\right\}_{m \in N_{0}}\right)}(\gamma+n+1, \lambda-\gamma)}{B(\gamma+1, \lambda-\gamma)} \frac{z^{n}}{n ! \Gamma(\alpha n+\alpha+\beta)} .
$$

In terms of (1.18), we obtain

$$
\frac{d}{d z} W_{\alpha, \beta, \gamma, \delta, \lambda, \sigma}^{\left(\left\{v_{m}\right\}_{m \in N_{0}}\right)}(z ; p)=\frac{\sigma \gamma}{\delta \lambda} W_{\alpha, \alpha+\beta, \gamma+1, \delta+1, \lambda+1, \sigma+1}^{\left(\left\{v_{m}\right\}_{m \in \mathbb{N}_{0}}\right)}(z ; p) .
$$

Similarly, continuing the process $(n-1)$ times, yields the desired result (3.11).

$$
\frac{d^{n}}{d z^{n}} W_{\alpha, \beta, \gamma, \delta, \lambda, \sigma}^{\left(\left\{v_{m}\right\}_{m \in N_{0}}\right)}(z ; p)=\frac{(\gamma)_{n}(\sigma)_{n}}{(\delta)_{n}(\lambda)_{n}} W_{\alpha, n \alpha+\beta, \gamma+n, \delta+n, \lambda+n, \sigma+n}^{\left(\left\{v_{m}\right\}_{m \in N_{0}}\right)}(z ; p) .
$$

Theorem 3.4. Let $p \in \mathbb{R}_{0}^{+}, x \in \mathbb{R}^{+}$. Also, let $r-2<\Re(\rho-\delta)<r-1$ for $m \in \mathbb{N}$. Then

$$
\begin{aligned}
\mathfrak{D}_{x ; c, d}^{\rho+1-\delta}\left(x^{\rho-1} W_{\alpha, \beta}^{\delta+r-1}(x)\right)= & \sum_{l=0}^{r}\left(\begin{array}{l}
r \\
l
\end{array}\right) \frac{\Gamma(\delta+r-1)}{\Gamma(\delta+l-1)} \frac{\Gamma(\rho+l)}{\Gamma(\delta+r+l-1)} \\
& \times x^{\delta+l-2} W_{\alpha, l \alpha+\beta}^{\rho+l, \delta+l+r-1 ; c, d}(x ; p), \quad(r \in \mathbb{N}) .
\end{aligned}
$$

Proof. Let $Q$ denote the left hand side of (3.13). Employing (3.6), we have

$$
Q=\frac{1}{\Gamma(r+\delta-\rho-1)} \times \frac{d^{r}}{d x^{r}} \int_{0}^{x} t^{\rho-1}(x-t)^{\delta+r-\rho-2} W_{\alpha, \beta}^{\delta+r-1}(t) \mathscr{F}\left[\left\{v_{m}\right\}_{m \in \mathbb{N}_{0}} ; \frac{-p x^{2}}{t(x-t)}\right] d t .
$$

Taking $t=u x$, we obtain

$$
Q=\frac{1}{\Gamma(r+\delta-\rho-1)}
$$




$$
\times \frac{d^{r}}{d x^{r}}\left\{x^{\delta+r-2} \int_{0}^{1} u^{\rho-1}(1-u)^{\delta+r-\rho-2} W_{\alpha, \beta}^{\delta+r-1}(u x) \mathscr{F}\left[\left\{v_{m}\right\}_{m \in \mathbb{N}_{0}} ; \frac{-p}{u(1-u)}\right] d u\right\} .
$$

Applying (2.1) in (3.14), we obtain

$$
Q=\frac{\Gamma(\rho)}{\Gamma(r+\delta-1)} \times \frac{d^{r}}{d x^{r}}\left\{x^{\delta+r-2} W_{\alpha, \beta}^{\rho, \delta+r-1 ; c, d}(x ; p)\right\} .
$$

By using the Leibniz's generalized rule for differentiation of product of two functions, we have

$$
Q=\frac{\Gamma(\rho)}{\Gamma(r+\delta-1)} \sum_{l=0}^{r}\left(\begin{array}{l}
r \\
l
\end{array}\right)\left\{\frac{d^{(r-l)}}{d x^{(r-l)}} x^{\delta+r-2}\right\}\left\{\frac{d^{l}}{d x^{l}} W_{\alpha, \beta}^{\rho, \delta+r-1 ; c, d}(x ; p)\right\} .
$$

Since we have

$$
\frac{d^{l}}{d x^{l}} x^{a}=\frac{\Gamma(a+1)}{a-l+1} x^{a-l} \quad\left(l \in \mathbb{N}_{o}\right)
$$

then, using (3.11) and in view of (3.16), we get the desired result (3.13).

By letting $r=1$, we get a particular case of Theorem 3.4 asserted by the following corollary:

Corollary 3.5. Let $p \in \mathbb{R}_{0}^{+}, x \in \mathbb{R}^{+}, \Re(\rho-\delta)>-1$ and $0<\Re(\rho)<\Re(\delta)$. Then, the undermentioned formula holds:

$$
\mathfrak{D}_{x ; \rho+1-\delta ; p}^{\left(\left\{v_{m}\right\}_{m \in \mathbb{N}_{0}}\right)}\left(x^{\rho-1} W_{\alpha, \beta}^{\delta}(x)\right)=\frac{\Gamma(\rho)}{\Gamma(\delta)} x^{\delta-2}\left\{(\delta-1) W_{\alpha, \beta ; \rho, \delta}^{\left\{v_{m}\right\}_{m \in N_{0}}}(x ; p)+\frac{\rho x}{\delta} W_{\alpha, \alpha+\beta ; \rho+1, \delta+1}^{\left\{v_{m}\right\}_{m \in N_{0}}}(x ; p)\right\} .
$$

Theorem 3.6. Let $\beta, \gamma, \delta, \sigma \in \mathbb{C} ; \alpha>-1, \Re(\delta)>\Re(\rho)>0, \Re(p)>0$. Then

$$
\begin{aligned}
& \left(\frac{d}{d z}\right)^{\kappa}\left[z^{\beta-1} W_{\alpha, \beta, \gamma, \delta, \lambda, \sigma}^{\left(\left\{v_{m}\right\}_{m \in \mathbb{N}_{0}}\right)}\left(\mu z^{\alpha} ; p\right)\right]=z^{\beta-\kappa-1} W_{\alpha, \beta-\kappa, \gamma, \delta, \lambda, \sigma}^{\left(\left\{v_{m}\right\}_{m \in \mathbb{N}_{0}}\right)}\left(\mu z^{\alpha} ; p\right) \\
& (\Re(\beta-\kappa)>0, \kappa \in \mathbb{N}) .
\end{aligned}
$$

Proof. Using (1.18), and employing term-wise differentiation $m$ times, we get

$$
\begin{aligned}
& \left(\frac{d}{d z}\right)^{\kappa}\left[z^{\beta-1} W_{\alpha, \beta, \gamma, \delta, \lambda, \sigma}^{\left(\left\{v_{m}\right\}_{m \in N_{0}}\right)}\left(\mu z^{\alpha} ; p\right)\right] \\
& =\sum_{n=0}^{\infty} \frac{B_{p}^{\left(\left\{v_{m}\right\}_{m \in N_{0}}\right)}(\gamma+n, \lambda-\gamma)}{B(\gamma, \lambda-\gamma)} \frac{(\mu)^{n}}{n ! \Gamma(\alpha n+\beta)} \frac{(\sigma)_{n}}{(\delta)_{n}}\left(\frac{d}{d z}\right)^{\kappa} z^{\alpha n+\beta-1} \\
& =\sum_{n=0}^{\infty} \frac{B_{p}^{\left(\left\{v_{m}\right\}_{m \in \mathbb{N}_{0}}\right)}(\gamma+n, \lambda-\gamma)}{B(\gamma, \lambda-\gamma)} \frac{(\sigma)_{n} \mu^{n}}{(\delta)_{n} \Gamma(\alpha n-\kappa+\beta)} \frac{z^{\alpha n-\kappa+\beta-1}}{n !} \\
& =z^{\beta-1-\kappa} \sum_{n=0}^{\infty} \frac{B_{p}^{\left(\left\{v_{m}\right\}_{m \in \mathbb{N}_{0}}\right)}(\gamma+n, \lambda-\gamma)}{\Gamma(\alpha n+\beta-\kappa)} \frac{z^{\alpha n} \mu^{n}}{n ! B(\gamma, \lambda-\gamma)} \frac{(\sigma)_{n}}{(\delta)_{n}} \\
& =z^{\beta-\kappa-1} W_{\alpha, \beta-\kappa, \gamma, \delta, \lambda, \sigma}^{\left(\left\{v_{m}\right\}_{m \in \mathbb{N}_{0}}\right)}\left(\mu z^{\alpha} ; p\right),
\end{aligned}
$$


which is the required result (3.18).

\section{Generating relation for the generalized Wright function}

In this section, we derive a generating relation involving the product of three generalized Wright functions. We recall the modified form of Exton's reult [6] given by

$$
\exp \left(s+t-\frac{z t}{s}\right)=\sum_{u=-\infty}^{\infty} \sum_{\nu=u *}^{\infty} s^{u} t^{v} \mathscr{H}_{v}^{u}(z)
$$

where,

$$
\mathscr{H}_{v}^{u}(z)={ }_{1} F_{1}(-v ; u+1 ; z) / u ! v !=\mathfrak{L}_{v}^{u}(z) /(u+v) !,
$$

and $\mathfrak{L}_{v}^{u}(z)$ denotes the Laguerre polynomials (see [10],[11],[20]) and $u *=\max (0,-u),(u=$ $0,1,2, \ldots)$.

Theorem 4.1. We derive the following partly bilateral and partly unilateral generating relation for the generalized Wright function

$$
\begin{aligned}
& W_{\alpha_{1}, \beta_{1}, \gamma_{1}, \delta_{1}, \lambda_{1}, \sigma_{1}}^{\left(\left\{v_{m_{1}} \xi_{m_{1} \in \mathbb{N}_{0}}\right)\right.}(s ; p) W_{\alpha_{2}, \beta_{2}, \gamma_{2}, \delta_{2}, \lambda_{2}, \sigma_{2}}^{\left(\left\{v_{m_{2}}\right\}_{m_{2} \in N_{0}}\right)}(t ; p) W_{\alpha_{3}, \beta_{3}, \gamma_{3}, \delta_{3}, \lambda_{3}, \sigma_{3}}^{\left(\left\{v_{m_{3}} m_{m_{3} \in \mathbb{N}_{0}}\right)\right.}\left(-\frac{z t}{s} ; p\right) \\
& =\sum_{u=-\infty}^{\infty} \sum_{\nu=u *}^{\infty} s^{u} t^{v}\left\{\begin{array}{l}
\alpha_{i}, \beta_{i}, \gamma_{i} \\
\delta_{i}, \lambda_{i}, \sigma_{i}
\end{array} \mathcal{H}_{\nu}^{u}(z)\right\} \quad(i=1,2,3),
\end{aligned}
$$

where

$$
\begin{aligned}
\left\{\begin{array}{c}
\alpha, \beta, \gamma \\
\delta, \lambda, \sigma
\end{array} \mathcal{H}_{v}^{u}(z)\right\}= & \sum_{u=-\infty}^{\infty} \sum_{\nu=u *}^{\infty} s^{u} t^{v} \times \sum_{k=0}^{\infty} \frac{B_{p}^{\left(\left\{v_{m_{3}}\right\}_{m_{3} \in \mathbb{N}_{0}}\right)}\left(\gamma_{3}+k, \lambda_{3}-\gamma_{3}\right)(-z)^{k}\left(\sigma_{3}\right)_{k}}{B\left(\gamma_{3}, \lambda_{3}-\gamma_{3}\right) k !\left(\beta_{3}\right)_{\alpha_{3} k}\left(\delta_{3}\right)_{k}} \\
& \times \frac{B_{p}^{\left(\left\{v_{m_{1}}\right\}_{m_{1} \in \mathbb{N}_{0}}\right)}\left(\gamma_{1}+u+k, \lambda_{1}-\gamma_{1}\right)\left(\sigma_{1}\right)_{u+k}}{B\left(\gamma_{1}, \lambda_{1}-\gamma_{1}\right)(u+k) !\left(\beta_{1}\right)_{\alpha_{1}(u+k)}\left(\delta_{1}\right)_{(u+k)}} \\
& \times \frac{B_{p}^{\left(\left\{v_{m_{2}}\right\}_{m_{2} \in \mathbb{N}_{0}}\right)}\left(\gamma_{2}+v-k, \lambda_{2}-\gamma_{2}\right)\left(\sigma_{2}\right)_{(\nu-k)}}{B\left(\gamma_{2}, \lambda_{2}-\gamma_{2}\right)(v-k) !\left(\beta_{2}\right)_{\alpha_{2}(\nu-k)}\left(\delta_{2}\right)_{(v-k)}} .
\end{aligned}
$$

Proof. Using the series expansions of (1.18), we write

$$
\begin{aligned}
M(z, s, t)= & \sum_{k=0}^{\infty} \frac{B_{p}^{\left(\left\{v_{m_{3}}\right\}_{m_{3} \in N_{0}}\right)}\left(\gamma_{3}+k, \lambda_{3}-\gamma_{3}\right)}{B\left(\gamma_{3}, \lambda_{3}-\gamma_{3}\right)} \frac{(-z)^{k}\left(\sigma_{3}\right)_{k}}{k ! \Gamma\left(\alpha_{3} k+\beta_{3}\right)\left(\delta_{3}\right)_{k}} \\
& \times \sum_{i=0}^{\infty} \frac{B_{p}^{\left(\left\{v_{m_{1}}\right\}_{m m_{1} \in N_{0}}\right)}\left(\gamma_{1}+i, \lambda_{1}-\gamma_{1}\right)}{B\left(\gamma_{1}, \lambda_{1}-\gamma_{1}\right)} \frac{s^{(i-k)}\left(\sigma_{1}\right)_{i}}{i ! \Gamma\left(\alpha_{1} i+\beta_{1}\right)\left(\delta_{1}\right)_{i}} \\
& \times \sum_{j=0}^{\infty} \frac{B_{p}^{\left(\left\{v_{m_{2}}\right\}_{m_{2} \in N_{0}}\right)}\left(\gamma_{2}+j, \lambda_{2}-\gamma_{2}\right) t^{(j+k)}\left(\sigma_{2}\right)_{j}}{B\left(\gamma_{2}, \lambda_{2}-\gamma_{2}\right) j ! \Gamma\left(\alpha_{2} j+\beta_{2}\right)\left(\delta_{2}\right)_{i}} .
\end{aligned}
$$


Replacing $i-k$ and $j+k$ by $u$ and $v$ respectively, and rearranging the series that is justified by the absolute convergence, yields

$$
\begin{gathered}
M(z, s, t)=\sum_{u=-\infty}^{\infty} \sum_{\nu=u *}^{\infty} \frac{s^{u} t^{\nu}}{\Gamma\left(\beta_{1}\right) \Gamma\left(\beta_{2}\right) \Gamma\left(\beta_{3}\right)} \times \sum_{k=0}^{\infty} \frac{B_{p}^{\left(\left\{v_{m_{3}}\right\}_{m_{3} \in N_{0}}\right)}\left(\gamma_{3}+k, \lambda_{3}-\gamma_{3}\right)(-z)^{k}\left(\sigma_{3}\right)_{k}}{B\left(\gamma_{3}, \lambda_{3}-\gamma_{3}\right) k !\left(\beta_{3}\right)_{\alpha_{3} k}+\left(\delta_{3}\right)_{k}} \\
\quad \times \frac{B_{p}^{\left(\left\{v_{m_{1}}\right\}_{\left.m_{1} \in \mathbb{N}_{0}\right)}\right.}\left(\gamma_{1}+u+k, \lambda_{1}-\gamma_{1}\right)\left(\sigma_{1}\right)_{u+k}}{B\left(\gamma_{1}, \lambda_{1}-\gamma_{1}\right)(u+k) !\left(\beta_{1}\right)_{\alpha_{1}(u+k)}\left(\delta_{1}\right)_{(u+k)}} \times \frac{B_{p}^{\left(\left\{v_{m_{2}}\right\}_{m_{2} \in \mathbb{N}_{0}}\right)}\left(\gamma_{2}+v-k, \lambda_{2}-\gamma_{2}\right)\left(\sigma_{2}\right)_{(\nu-k)}}{B\left(\gamma_{2}, \lambda_{2}-\gamma_{2}\right)(\nu-k) !\left(\beta_{2}\right)_{\alpha_{2}(\nu-k)}\left(\delta_{2}\right)_{(\nu-k)}} .
\end{gathered}
$$

\section{References}

[1] A. Belafhal, N. Nossir, L. Dalil-Essakali and T. Usman, AIMS Mathematics, 5 (2020), 1260-1274.

[2] M. A. Chaudhry, A. Qadir, H. M. Srivastava and R. B. Paris, Extended hypergeometric and confluent hypergeometric functions, Appl. Math. Comput., 159 (2004), 589-602.

[3] P. Agarwal, J. Choi and R. B. Paris, Extended Riemann-Liouville fractional derivative operator and its applications, Journal of Nonlinear Science and Applications, 8 (2015), 451-466.

[4] A. Erdélyi, W. Magnus, F. Oberhettinger and F. Tricomi. Higher transcendental functions, (California institute of technology h. Bateman ms project), 2 (1953).

[5] M. El-Shahed and A. Salem, An extension of Wright function and its properties, Hindawi Publishing Corporation, Journal of Mathematics, Article ID 950728 (2015), 11 pages.

[6] H. Exton, A new generating function for the associated Laguerre polynomials and resulting expansions, Jñañabha, 13 (1983), 143-149.

[7] H. G. Flegg, Mikusinski's Operational Calculus, International Journal of Mathematical Education in Science and Technology, 5 (1974), 131-137.

[8] R. Goreno, Y. Luchko and F. Mainardi, Analytical properties and applications of the Wright function, Fractional Calculus and Applied Analysis, 2, (1999), 383-414.

[9] P. Karlsson and H. Srivastava, Multiple gaussian hypergeometric series, In Ellis Horwood Series: Mathematics and its Applications. Halsted Press, Wiley New York, 1985.

[10] N. U. Khan, M. M. Eltikali, M. Kamarujjama and T. M. Elfrgani, Some unified integrals of Mittag Leffler functions, Palestine Journal of Mathematics, 7 (2018), 115-120.

[11] N. U. Khan and M. Ghayasuddin, Some generating relations of extended Mittag-Leffler functions, Kyungpook Mathematical Journal, 59 (2019), 325-333.

[12] N. U. Khan, T. Usman and M. Aman, Extended Beta, Hypergeometric and confluent Hypergeometric functions, Trans. Natl. Acad. Sci. Azerb. Ser. Phys.-Tech. Math. Sci., 39 (2019), 1-16.

[13] N. U. Khan and M. Ghayasuddin, Generalization of extended appell's lauricella's hypergeometric functions, Honam Mathematical Journal, 37 (2015), 113-126.

[14] V. Kiryakova, Some special functions related to fractional calculus and fractional (non-integer) order control systems and equations, Facta Universitatis Series: Automatic Control and Robotics, 7 (2008), 79-98.

[15] F. Mainardi and G. Pagnini, The role of the Fox-Wright functions in fractional sub-diffusion of distributed order, Journal of Computational and Applied Mathematics, 207 (2007), 245-257.

[16] E. Özergin, M. A. Özarslan and A. Altin, Extension of gamma, beta and hypergeometric functions, Journal of Computational and Applied Mathematics, 235 (2011), 4601-4610.

[17] H. M. Srivastava, R. Agarwal and S. Jain, Integral transform and fractional derivative formulas involving the extended generalized hypergeometric functions and probability distributions, Mathematical Methods in the Applied Sciences, 40 (2017), 255-273. 
[18] H. M. Srivastava and J. Choi, Zeta and $q$-Zeta Functions and Associated Series and Integrals, Elsevier Science Publishers, Amsterdam, London and New York, 2012.

[19] H. M. Srivastava, R. K. Parmar and P. Chopra, A class of extended fractional derivative operators and associated generating relations involving hypergeometric functions, AXIOMS, 1 (2012), 238-258.

[20] G. Szegö, Orthogonal Polynomials, American Mathematical Society Colloquium Publications, American Mathematical Society: Providence, Rhode Island, 23, 1975.

[21] E. M. Wright, On the coefficients of power series having exponential singularities, Journal of the London Mathematical Society, s1-8 (1933), 71-79.

[22] E. M. Wright, The asymptotic expansion of the generalized hypergeometric function, J. London. Math. Soc., 10 (1935), 286-293.

[23] E. M. Wright, The asymptotic expansion of integral functions defined by Taylor Series, Philos. Trans. Roy. Soc. London, 238 (1940), 423-451.

[24] E. M. Wright, The asymptotic expansion of the generalized hypergeometric function II, Proc. London. Math. Soc., 46 (1935), 389-408.

Department of Applied Mathematics, Faculty of Engineering and Technology, Aligarh Muslim University, Aligarh202002, India.

E-mail: nukhanmath@gmail.com

E-mail: talhausman.maths@gmail.com

E-mail: mohdaman.maths@gmail.com 